\title{
PATRONES DE VARIACIÓN Y DISTRIBUCIÓN GEOGRÁFICA EN FENOTIPOS QUÍMICOS FOLIARES DE Persea americana var. drymifolia
}

\section{PATTERNS OF VARIATION AND GEOGRAPHIC DISTRIBUTION IN FOLIAR CHEMICAL PHENOTYPES OF Persea americana var. drymifolia}

\author{
Guadalupe Torres-Gurrola $^{1^{*}, \text { Salvador Montes-Hernández }}{ }^{2}$ y Francisco J. Espinosa-García ${ }^{1}$
}

\begin{abstract}
${ }^{1}$ Centro de Investigaciones en Ecosistemas, Universidad Nacional Autónoma de México. Antigua carretera a Pátzcuaro No. 8701, Col. ex-Hacienda de San José de la Huerta. 58190, Morelia, Mich. Tel. 01-443-322-27-77 Ext. 42628, Fax 01-443-322-27-19. ${ }^{2}$ Campo Experimental Bajío, Instituto Nacional de Investigaciones Forestales, Agrícolas y Pecuarias. Km. 6.5 carretera Celaya-San Miguel de Allende. Celaya, Gto.
\end{abstract}

*Autor para correspondencia (gtorres@oikos.unam.mx)

\section{RESUMEN}

Persea americana var. drymifolia (aguacate criollo) en forma silvestre se encuentra en bosques con un precario estado de conservación. Se estudió la química foliar de poblaciones de este aguacate ubicadas en el Banco de Germoplasma del Instituto Nacional de Investigaciones Forestales, Agrícolas y Pecuarias en Celaya, Guanajuato, uno de los bancos más representativos para esta variedad en México. Se tomaron hojas maduras de 291 árboles correspondientes a 35 accesiones de siete estados del país. Se determinó el perfil químico foliar por cromatografía de gases acoplada a espectrometría de masas. Se cuantificó la concentración de 33 compuestos, de los cuales se identificaron 28. Los más abundantes fueron estragol y cariofileno; cada uno representó más de $10 \%$ de la concentración total de la muestra. La variación química de los árboles clasificada con análisis de conglomerados, mostró grupos y subgrupos con 40 a $60 \%$ de similitud. Los subgrupos químicos que contienen a la mayoría de los árboles a $40 \%$ de similitud se diferenciaron entre sí principalmente por estragol, sabineno y $\beta$-cubebeno. Estragol, cariofileno, pcimeno, $\beta$-felandreno y chavicol-metil-éter fueron los componentes característicos en otros subgrupos a una escala de similitud de $60 \%$. Ningún subgrupo se asoció con una distribución geográfica particular. Se encontró mayor variación entre estados que dentro de ellos, para la mayoría de los compuestos químicos.

Palabras clave: Persea americana var. drymifolia, cariofileno, estragol, variación fitoquímica.

\section{SUMMARY}

Wild Persea americana var. drymifolia (creole avocado) is found in forests with a precarious conservation state. The foliar chemistry of creole avocados from the Germoplasm Bank of the Instituto Nacional de Investigaciones Forestales, Agrícolas y Pecuarias in Celaya, Guanajuato, one of the most representative banks for this avocado variety in México, was estudied. We sampled 291 trees belonging to 35 accessions from seven Mexican states. The foliar chemical profile was determined by gas chromatography coupled to a mass spectrometer. We found and quantified the concentration of 33 compounds, out of which 28 were identified. The most abundant compounds were estragole and caryophyllene, each representing over 10
$\%$ of the total concentration in the sample. The chemical variation of the trees was classified by a cluster analysis in which groups and sub-groups with 40 to $60 \%$ of similarity were formed. The chemical subgroups containing most of the trees at $40 \%$ of similarity were differentiated mainly by estragole, sabinene and $\beta$-cubebene. Estragole, caryophyllene, p-cimene $\beta$-phellandrene and chavicolmethyl-ether were the characteristic components in other sub-groups with $60 \%$ of similarity. A greater variation was found between the states of México than within them, for most chemical compounds.

Index words: Persea americana var. drymifolia, caryophyllene, estragole, phytochemical variation.

\section{INTRODUCCIÓN}

El aguacate es originario de las áreas montañosas del centro y este de México, y de partes altas de Guatemala (Williams, 1977), de donde se ha llevado al resto del mundo (Barrientos-Priego y López-López, 1998). Se reconocen tres razas: Mexicana (Persea americana var. drymifolia), Guatemalteca ( $P$. americana var. guatemalensis) y Antillana ( $P$. americana var. americana). La variedad drymifolia produce frutos que se consumen y comercializan localmente y se usa como pie de injerto para el cultivar 'Hass'. Esta variedad también es importante, pues junto con $P$. americana var. guatemalensis son los progenitores del cultivar 'Hass', el más distribuido en el mundo (Fielder et al., 1998).

Persea americana var. drymifolia (aguacate criollo) en forma silvestre se encuentra en bosques mesófilos de montaña y de Lauraceas (Challenger, 1998; LoreaHernández, 2002) cuyo estado de conservación es muy precario, y cuya superficie original ha disminuido por la apertura de nuevas áreas a la agricultura y la ganadería, sobrepastoreo, incendios forestales, avance de las áreas 
urbanas y la explotación maderera (Challenger, 1998; Lorea-Hernández, 2002; Sánchez-Pérez, 1999). Así, una parte considerable de la enorme riqueza fitogenética de Persea spp. está amenazada por la destrucción de estos ecosistemas y por la sustitución de cultivares tradicionales por cultivares mejorados.

Una manera de rescatar esta riqueza es a través del establecimiento de bancos de germoplasma; varias instituciones mexicanas se han dado a la tarea de conservar, evaluar y documentar los recursos genéticos del aguacate en estos bancos; entre éstos se encuentra el del Instituto Nacional de Investigaciones Forestales, Agrícolas y Pecuarias (INIFAP) en Celaya, México, que contiene aproximadamente $38.5 \%$ del total de las accesiones registradas de diferentes instituciones mexicanas (BarrientosPriego y López-López, 1998). Sin embargo, el estudio y la caracterización de la variación química del aguacate criollo y del cv. 'Hass' (en bancos de germoplasma o en forma silvestre) han tenido poca atención (Bergh et al., 1973; Espinosa-García et al., 2001; Rincón-Hernández et $a l ., 2005)$, a pesar de que poseen metabolitos secundarios asociados con la resistencia de las plantas contra plagas y enfermedades (Edwards et al., 1993; Espinosa-García, 2001; Langenheim, 2003). Muchos de estos compuestos (p. e., terpenoides) están bajo un fuerte control genético, y pueden ser usados como marcadores para la resistencia contra plagas y patógenos (Lavi et al., 1993a, b; Mhameed et al., 1995). La variabilidad química en plantas frecuentemente está asociada con la distribución geográfica (Azevedo et al., 2001; Dodd y Rafii, 1994; Goralka y Langenheim, 1995), por lo que es posible que esta asociación también se encuentre en $P$. americana var. drymifolia.

La var. drymifolia es rica en metabolitos secundarios antagónicos contra herbívoros y patógenos, particularmente en terpenoides y fenilpropanoides de los aceites esenciales foliares (Rincón-Hernández et al., 2005; Sagrero-Nieves y Bartley, 1995), aunque otros compuestos activos también se han encontrado en otros tejidos de la planta (Prabha et al., 1980).

El banco de germoplasma de $P$. americana var. drymifolia del INIFAP en Celaya, Gto., es uno de los bancos con mejor representación de Persea americana var. drymifolia con cerca de 35 accesiones provenientes de nueve estados de México (Barrientos-Priego y López-López, 1998), y su variabilidad genética o química no ha sido estudiada.

Los objetivos de este trabajo fueron: a) Describir la variación de terpenoides y fenilpropanoides volátiles foliares en las accesiones de $P$. americana var. drymifolia del banco de germoplasma del INIFAP-Celaya; y b) Detectar si la distribución geográfica del aguacatero criollo está asociada con su composición química foliar.

\section{MATERIALES Y MÉTODOS}

Se tomaron hojas de las accesiones del banco de Germoplasma del Campo Experimental Bajío, del INIFAP, en Celaya, Gto. $\left(20^{\circ} 26^{\prime} \mathrm{LN}\right.$ y $103^{\circ} 19^{\prime} \mathrm{LO}$, a 1750 $\mathrm{msnm})$. En este banco se encuentran entre 1 y 55 árboles de cada una de las accesiones (mediana $=4$ ) de $P$. americana var. drymifolia, de entre 30 y 33 años de edad. El sitio tiene clima semicálido subhúmedo, con precipitación media de 600 a $800 \mathrm{~mm}$, y temperatura media entre 18 y $20{ }^{\circ} \mathrm{C}$ (García, 1988). En el suelo predominan vertisoles pélicos de alta fertilidad (FAO-UNESCO, 1970).

El material analizado procede de accesiones de: Celaya, Comonfort, y San Miguel de Allende, en el Edo. de Guanajuato; Tenancingo, Edo. de México; Uruapan y Peribán de Ramos, del Edo. de Michoacán; Tetela del Volcán, del Edo. de Morelos; General Terán, del Edo. de Nuevo León; Villa Corregidora, del Edo. de Querétaro; Coscomatepec y Calcahualco, del Edo. de Veracruz.

\section{Toma de muestras}

Se tomaron hojas de 291 árboles provenientes de 35 accesiones de aguacate criollo. Se colectaron dos hojas maduras de cada árbol para determinar su perfil químico. Las colectas se realizaron entre el 11 de junio y el 14 de julio del 2003. Las hojas se colocaron en una bolsa de plástico y transportadas en hielo seco de inmediato al laboratorio para el análisis químico.

\section{Procedimiento de extracción y cuantificación de los metabolitos secundarios}

Las hojas se dividieron a lo largo de la vena central; una parte se deshidrató en estufa a $80{ }^{\circ} \mathrm{C}$ por $24 \mathrm{~h}$ para determinar su peso seco, y la otra mitad se pesó y se maceró en un frasco ámbar de $75 \mathrm{~mL}$ con hexano durante una semana. Después, la hoja macerada en hexano se trituró con nitrógeno líquido, se añadió hexano y un $1 \mathrm{mg}$ de tetradecano disuelto en hexano como estándar interno. Después de triturar de nuevo, el extracto se filtró y secó con sulfato de sodio anhidro. Se adicionó pirogalol como antioxidante (Sigma-Aldrich ${ }^{\circledR}$ ). El extracto se concentró con una corriente suave de $\mathrm{N}_{2}$ hasta $1 \mathrm{~mL}$, y permaneció a $-20{ }^{\circ} \mathrm{C}$ hasta su análisis.

Las muestras se inyectaron (1 a $2 \mu \mathrm{L})$ con inyección dividida 60:1 en un cromatógrafo de gases (Agilent HP6890®), provisto con detector de ionización de flama 
(FID) y una columna capilar HP 5 (fenil metil-siloxano) de $30 \mathrm{~m}$, con un diámetro interno de $0.25 \mathrm{~mm}$ y $0.25 \mu \mathrm{m}$ de recubrimiento. Se usó helio como gas acarreador; inyector a $250{ }^{\circ} \mathrm{C}$; presión en el inyector, $86.9 \mathrm{kPa}$; flujo en el inyector, $67.2 \mathrm{~mL} \mathrm{~min}^{-1}$; flujo de helio en la columna, $1.0 \mathrm{~mL} \mathrm{~min}^{-1}$; presión de helio en la columna, 86.7 $\mathrm{kPa}$, con una velocidad promedio de $26 \mathrm{~cm} \mathrm{~s}^{-1}$. El programa de temperatura para el horno fue: temperatura inicial $50{ }^{\circ} \mathrm{C}$, incremento de $20{ }^{\circ} \mathrm{C} \mathrm{min}^{-1}$ hasta llegar a 200 ${ }^{\circ} \mathrm{C}$; aumento de $15{ }^{\circ} \mathrm{C} \mathrm{min}{ }^{-1}$ hasta llegar a $280{ }^{\circ} \mathrm{C}$ y aumento de $20{ }^{\circ} \mathrm{C} \mathrm{min}^{-1}$ hasta llegar a $300{ }^{\circ} \mathrm{C}$.

Algunas muestras fueron analizadas en un cromatógrafo de gases (Agilent HP6890) acoplado a espectrómetro de masas (Agilent 6890®), equipado con la misma columna usada en FID para determinar la composición química de los picos cromatográficos. Se usó helio como gas acarreador y las siguientes condiciones: inyector a 250 ${ }^{\circ} \mathrm{C}$; presión en el inyector, $8.5 \mathrm{kPa}$; flujo en el inyector, $63.3 \mathrm{~mL} \mathrm{~min}^{-1}$; flujo de helio en la columna, $0.5 \mathrm{~mL} \mathrm{~min}$ ${ }^{1}$; presión de helio en la columna, $8.5 \mathrm{kPa}$, con una velocidad promedio de $26 \mathrm{~cm} \mathrm{~s}^{-1}$. El programa de temperatura para el horno fue el mismo que se usó para las corridas con FID.

\section{Identificación y cuantificación de los compuestos químicos}

Los compuestos químicos se identificaron con la biblioteca de espectros de masas NIST 98. Para confirmar las identificaciones se usaron tiempos de retención y espectros de masas de compuestos puros (Sigma-Aldrich ${ }^{\circledR}$ ): linalol, $\alpha$-cubebeno, $\alpha$-pineno, $\beta$-pineno, $\beta$-mirceno, anetol, camfeno, nerolidol, $\beta$-cariofileno, limoneno, $\alpha$ humuleno, y $\gamma$-terpineol (Rincón-Hernández y EspinosaGarcía, 2008). Se verificó la pureza de cada pico mediante el programa MSD ChemStation (Agilent Technologies $\left.{ }^{\circledR}\right)$ y se nombraron sólo los compuestos químicos cuyo espectro registró como mínimo $90 \%$ de concordancia con el espectro de la biblioteca NIST 98. Para la cuantificación de los compuestos químicos se hizo una comparación entre la altura del pico del analito y el estándar interno. Se hizo una matriz de concentración de los compuestos químicos identificados ( $\mathrm{mg} \mathrm{g}^{-1}$ de hoja seca).

\section{Análisis estadístico}

Se efectuaron dos matrices; una de concentración (mg $\mathrm{g}^{-1}$ de hoja seca del compuesto) y otra de proporciones; esta última expresa la concentración de cada compuesto químico en relación con la concentración total de todos los compuestos presentes en la muestra. En el análisis de agrupamiento se generó un dendrograma con el algoritmo del método de pares de grupos no ponderados con promedio aritmético (UPGMA) y distancias de Manhattan. Para la caracterización química de los árboles se hizo una descripción jerárquica de las diferencias químicas entre los grupos obtenidos; se tomaron en cuenta sólo los compuestos con representación mayor a $1 \% \pm$ error estándar.

La composición de la muestra se comparó con cantidades relativas de los compuestos en lugar de sus cantidades absolutas, para evitar confundir factores ambientales y genéticos que pueden influir en la producción de los compuestos químicos (White y Nilsson, 1984). Se agruparon a los individuos de las localidades por estado en "poblaciones" con los datos de latitud, longitud y altitud; las "poblaciones" se encuentran separadas por al menos $3700 \mathrm{~m}$ o por $100 \mathrm{~m}$ de altitud. Algunas "poblaciones" son homónimas, por lo que se asignaron números progresivos de acuerdo con sus coordenadas geográficas. Se usaron los datos de la matriz de proporciones en el análisis de varianza anidado, análisis discriminantes y regresión múltiple.

\section{RESULTADOS Y DISCUSIÓN}

En total se estudiaron 18 localidades definidas de acuerdo con sus coordenadas geográficas y el número de individuos de cada población (Cuadro 1). Se encontraron 33 compuestos químicos en la fracción volátil de las hojas de $P$. americana var. drymifolia; 28 de ellos fueron identificados (Cuadro 2): 10 monoterpenoides (1S- $\alpha$-pineno, L- $\beta$-pineno, sabineno, $\beta$-pineno, $\alpha$-felandreno, $p$-cimeno, $1 \mathrm{R}$ - $\alpha$-pineno, eucaliptol, cis- $\beta$-terpineol y $\beta$-linalol); siete sesquiterpenoides ( $\beta$-cubebeno, $\alpha$-humuleno, cariofileno, óxido de cariofileno, germacreno $\mathrm{D}$, nerolidol y germacreno D-4-ol); seis fenilpropanoides (eugenol, eugenolmetil-éter, estragol, anetol, chavicol y chavicol-metiléter); un acetato (fenol-4-(2-propenil)-acetato) y cuatro alcanos (5-metil-tridecano, octadecano, hexadecano y heptadecano).

La cantidad relativa promedio de la mayoría de los compuestos fue igual o menor a $2 \%$ (Cuadro 2). Estragol fue el compuesto predominante con $38 \%$ seguido por cariofileno con $11 \%$; $p$-cimeno, sabineno, $\beta$-pineno, anetol, eugenol-metil-éter, germacreno $\mathrm{D}$, hexadecano y óxido de cariofileno se encontraron entre $2 \%$ y $4 \%$. 1R- $\alpha$-pineno, eucaliptol, $\beta$-linalol, chavicol, 5-metil-tridecano, fenol-4(2-propenil)-acetato, 1c.n.i.(7.14) (1 compuesto no identificado(tiempo de retención promedio); 2c.n.i.(7.9) y 2c.n.i.(8.11), son compuestos que se encontraron en proporciones menores de $1 \%$ por lo que únicamente se tomaron en cuenta para el análisis de agrupamiento. 
Cuadro 1. Ubicación de las localidades y número de muestras en cada población de los árboles de P. americana var. drymifolia del Banco de Germoplasma del INIFAP en Celaya, Guanajuato.

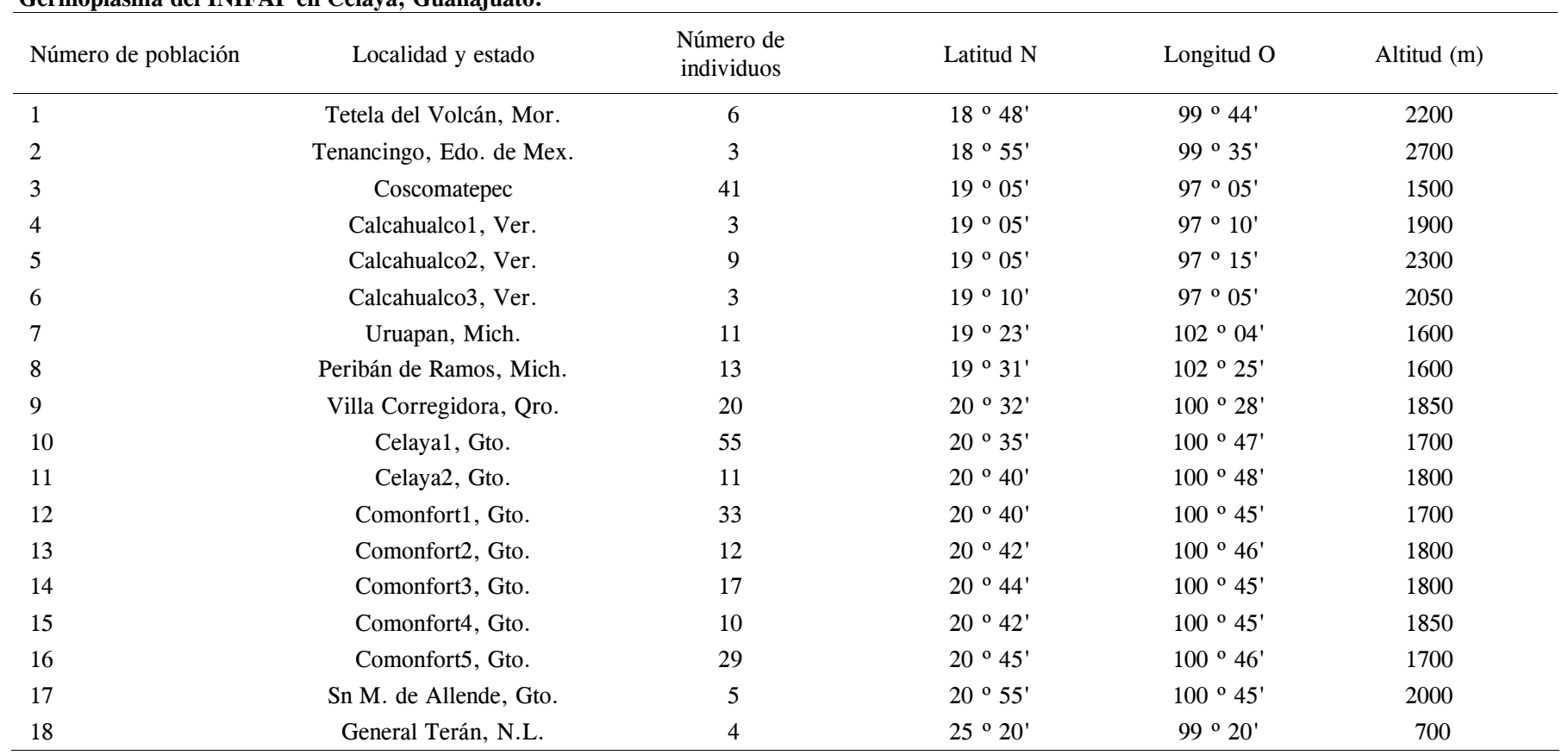

\section{Grupos y subgrupos del banco de germoplasma}

En el dendrograma se formaron dos grupos principales que no se parecen entre sí (Figura 1). El Grupo I solo contiene $1 \%$ de los árboles y el Grupo II al resto, y éste está integrado por dos subgrupos: el IIA con $1 \%$, y el IIB con $98 \%$, y éste está integrado a su vez por dos subsubgrupos: IIB. 1 que representa $49 \%$, y IIB. 2 con $49 \%$; estos fueron los sub-subgrupos principales, con diferencias entre sí de $60 \%$.

El sub-subgrupo IIB1 está integrado por varios conjuntos que corresponden a $2 \%$ de los árboles, además de dos grandes sub-conjuntos (IIB1b2.1 y IIB1b2.2, cada uno representa $23 \%$ ), que son diferentes entre sí en $40 \%$. Al sub-subgrupo IIB2 lo integran los conjuntos IIB2.a y IIB2.b que tienen $1 \%$ y $48 \%$, respectivamente, los cuales difieren en $50 \%$; el conjunto IIB2.b está integrado por los subconjuntos IIB2b.1 y IIB2b. 2 que tienen $18 \%$ y $31 \%$, los cuales son diferentes entre sí en $20 \%$. A una escala de diferencia de $10 \%$ se obtuvieron 47 grupos de fenotipos químicos entre todas las muestras.

Grupos generados por características químicas. En el análisis discriminante (Cuadro 3), los compuestos que caracterizan a los sub-subgrupos IIB. 1 y IIB. 2 son: estragol; 3c.n.i. (7.77); $\beta$-cubebeno y sabineno. Estragol es el compuesto más característico, pues el primer subsubgrupo contiene la cuarta parte de lo que tiene el segundo sub-subgrupo. En los subconjuntos IIB2b.1 y IIB2b.2, estragol es el compuesto que los diferencia; en el segundo subconjunto su proporción es $22 \%$ mayor que la del primero. En los subconjuntos IIB1b2.1 y IIB1b2.2, el compuesto que los diferencia también es estragol, que es nueve veces mayor en el primer subconjunto que en el segundo; cariofileno y p-cimeno están a la mitad en el primer subconjunto que en el segundo.

\section{Distribución geográfica del origen de las accesiones según su grupo químico}

Según su grupo químico, las accesiones tienen una distribución geográfica muy similar (subgrupos IIB.1 y IIB.2), (Figura 2). Para los subconjuntos (IIB1b2.1, IIB1b2.2) que incluyen $49 \%$ de los árboles en la escala de disimilitud de $40 \%$, tampoco hubo distribución geográfica diferencial, excepto para las accesiones de Nuevo León que pertenecen al subconjunto IIB1b2.1 (Figura 3). Con la escala de disimilitud de $20 \%$, en los subconjuntos IIB2b.1 y IIB2b.2 nuevamente se encontró una distribución similar, excepto para Nuevo León donde sólo el último subconjunto tiene representantes (Figura 4). En general, la distribución geográfica de las accesiones fue muy similar en escalas de disimilitud mayores a $20 \%$. 
Cuadro 2. Compuestos volátiles foliares más abundantes en los árboles de $P$. americana var. drymifolia ubicados en el Banco de Germoplasma INIFAP en Celaya, Guanajuato. Análisis de varianza anidado de compuestos entre y dentro de los estados. Los valores entre paréntesis representan el porcentaje de la varianza explicada.

\begin{tabular}{|c|c|c|c|c|c|}
\hline \multirow[b]{2}{*}{ Compuesto } & \multirow{2}{*}{$\begin{array}{l}\text { Mediana del } \\
\text { tiempo de re- } \\
\text { tención (min) }\end{array}$} & \multirow{2}{*}{$\begin{array}{l}\text { Porcentaje medio } \\
\text { del compuesto } \\
\text { (por g de hoja } \\
\text { seca) }\end{array}$} & \multicolumn{3}{|c|}{ Media de suma de cuadrados } \\
\hline & & & Entre estados & $\begin{array}{l}\text { Dentro de } \\
\text { estados }\end{array}$ & $\begin{array}{c}\text { Error estándar (y } \\
\text { porcentaje de varianza) }\end{array}$ \\
\hline L- $\beta$-pineno & 3.6 & $2.0 \pm 0.2$ & $6.6(42)$ & $4.0(26)$ & $5.0(32)$ \\
\hline sabineno & 3.8 & $2.4 \pm 0.2$ & $1.2(19)$ & $2.3(38)$ & $2.7(43)$ \\
\hline$\beta$-pineno & 3.8 & $3.5 \pm 0.3$ & $19.1(71)^{* * *}$ & $5.4(20)^{* * *}$ & $2.2(08)$ \\
\hline$\alpha$-felandreno & 3.9 & $0.4 \pm 0.1$ & $1.1(47)$ & $0.6(27)$ & $0.6(26)$ \\
\hline eucaliptol & 4.1 & $0.5 \pm 0.1$ & ------------- & - & 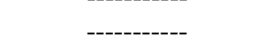 \\
\hline cis- $\beta$-terpineol & 4.3 & $1.2 \pm 0.1$ & $1.6(66)^{* * *}$ & $0.5(23)^{* *}$ & $0.3(12)$ \\
\hline$\beta$-linalool & 4.7 & $0.4 \pm 0.1$ & ------------ & ----------------- & ---------- \\
\hline 1 c.n.i.(5.02) & 5.0 & $0.9 \pm 0.2$ & $10.7(54)^{*}$ & $5.7(28)^{*}$ & $3.6(18)$ \\
\hline estragol & 5.4 & $38.3 \pm 1.3$ & $3255.7(64) * *$ & $1130.3(22)^{*}$ & $725.9(14)$ \\
\hline chavicol & 5.8 & $0.1 \pm<0.1$ & --- & 11.0.0 & 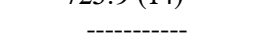 \\
\hline fenol-4-(2-propenyl)- acetato & 7.0 & $0.8 \pm 0.1$ & -10.0 (11) & $20 .+(21)$ & - n \\
\hline$\beta$-cubebeno & 7.0 & $1.0 \pm 0.4$ & $42.0(32)$ & $31.8(24)$ & $57.2(44)$ \\
\hline cariofileno & 7.1 & $11.2 \pm 0.3$ & $6.7(11)$ & $34.5(54)^{*}$ & $22.5(35)$ \\
\hline octadecano & 7.1 & $1.7 \pm 0.2$ & $2.0(66)^{* * *}$ & $0.8(27)^{* * *}$ & $0.2(7)$ \\
\hline 1 c.n.i.(7.14) & 7.1 & $0.5 \pm 0.1$ & 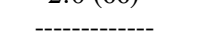 & 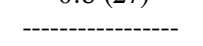 & - - - \\
\hline eugenol & 7.2 & $0.3 \pm 0.2$ & $<0.1(0.4)$ & $1.5(21)$ & $5.6(78)$ \\
\hline$\alpha$-humuleno & 7.3 & $1.7 \pm 0.1$ & $0.2(38)$ & $<0.1(27)$ & $0.2(35)$ \\
\hline germacreno D & 7.4 & $3.1 \pm 0.2$ & $1.0(48)$ & $0.6(28)$ & $0.5(24)$ \\
\hline 3 c.n.i. $(7.77)$ & 7.8 & $1.2 \pm 0.1$ & $0.1(28)$ & $0.1(26)$ & $0.2(47)$ \\
\hline nerolidol & 7.8 & $1.3 \pm 0.2$ & $1.9(30)$ & $3.1(49)^{*}$ & $1.3(21)$ \\
\hline hexadecano & 7.9 & $3.8 \pm 0.3$ & $1.8(10)$ & $10.6(56)^{* * *}$ & $6.7(35)$ \\
\hline 2 c.n.i.(7.9) & 7.9 & $0.7 \pm 0.1$ & ------------ & ----------------- & ---------- \\
\hline 2 c.n.i. $(8.11)$ & 8.1 & $0.8 \pm 0.1$ & ------------- & ------------------ & ----------- \\
\hline
\end{tabular}

Composición cuantitativa (\% de total de compuestos); $\mathrm{n}=291$; (c.n.i.(T.R.P.)) $=$ compuesto no identificado(tiempo de retención promedio); ----- $=$ compuestos que no se consideraron en el análisis; *,**,***; Significativo a $\mathrm{P} \leq 0.05, \mathrm{P} \leq 0.01$, y $\mathrm{P} \leq 0.001$

Cuadro 3. Resultado del análisis de discriminantes que se hizo en cada grupo de árboles de $\boldsymbol{P}$. americana var. drymifolia del Banco de Germoplasma INIFAP en Celaya, Guanajuato. Solamente se registraron los compuestos que diferenciaron a cada grupo.

\begin{tabular}{|c|c|c|c|c|c|}
\hline Compuestos & Media & Media & Error estándar & $\mathrm{F}$ & Significancia \\
\hline & Grupo IIB.1 & Grupo IIB.2 & & & \\
\hline estragol & 18.5 & $69.2 * * *$ & 1.2 & 620.1 & $* * *$ \\
\hline 3 c.n.i.(7.77) & 0.2 & $0.1 * * *$ & $<0.1$ & 11.7 & $* * *$ \\
\hline$\beta$-cubebeno & $<0.1$ & $0.5 *$ & 0.3 & 5.6 & $*$ \\
\hline \multirow[t]{2}{*}{ sabineno } & 0.9 & $0.5 *$ & 0.1 & 4.0 & $*$ \\
\hline & Grupo IIB2b.1 & Grupo IIB2b.2 & & & \\
\hline \multirow[t]{2}{*}{ estragol } & 59.1 & 75.6 & $0.8 ; 0.6$ & 259.9 & $* * *$ \\
\hline & Grupo IIB1b2.1 & Grupo IIB1b2.2 & & & \\
\hline estragol & 34.9 & 3.6 & 1.0 & 562.3 & $* * *$ \\
\hline cariofileno & 4.0 & 6.6 & 0.5 & 6.9 & $* *$ \\
\hline p-cimeno & 1.7 & 3.3 & 0.4 & 5.6 & $*$ \\
\hline$\alpha$-felandreno & 0.1 & 0.3 & 0.1 & 4.1 & $*$ \\
\hline 1 c.n.i.(5.02) & 0.4 & 0.4 & 0.1 & 6.4 & * \\
\hline chavicol-metil-éter & $<0.1$ & $<0.1$ & $<0.1$ & 4.1 & $*$ \\
\hline
\end{tabular}

(c.n.i.) $=$ Compuesto no identificado, $*, * *, * * *$; Significativo a $\mathrm{P} \leq 0.05, \mathrm{P} \leq 0.01$, y $\mathrm{P} \leq 0.001$. 


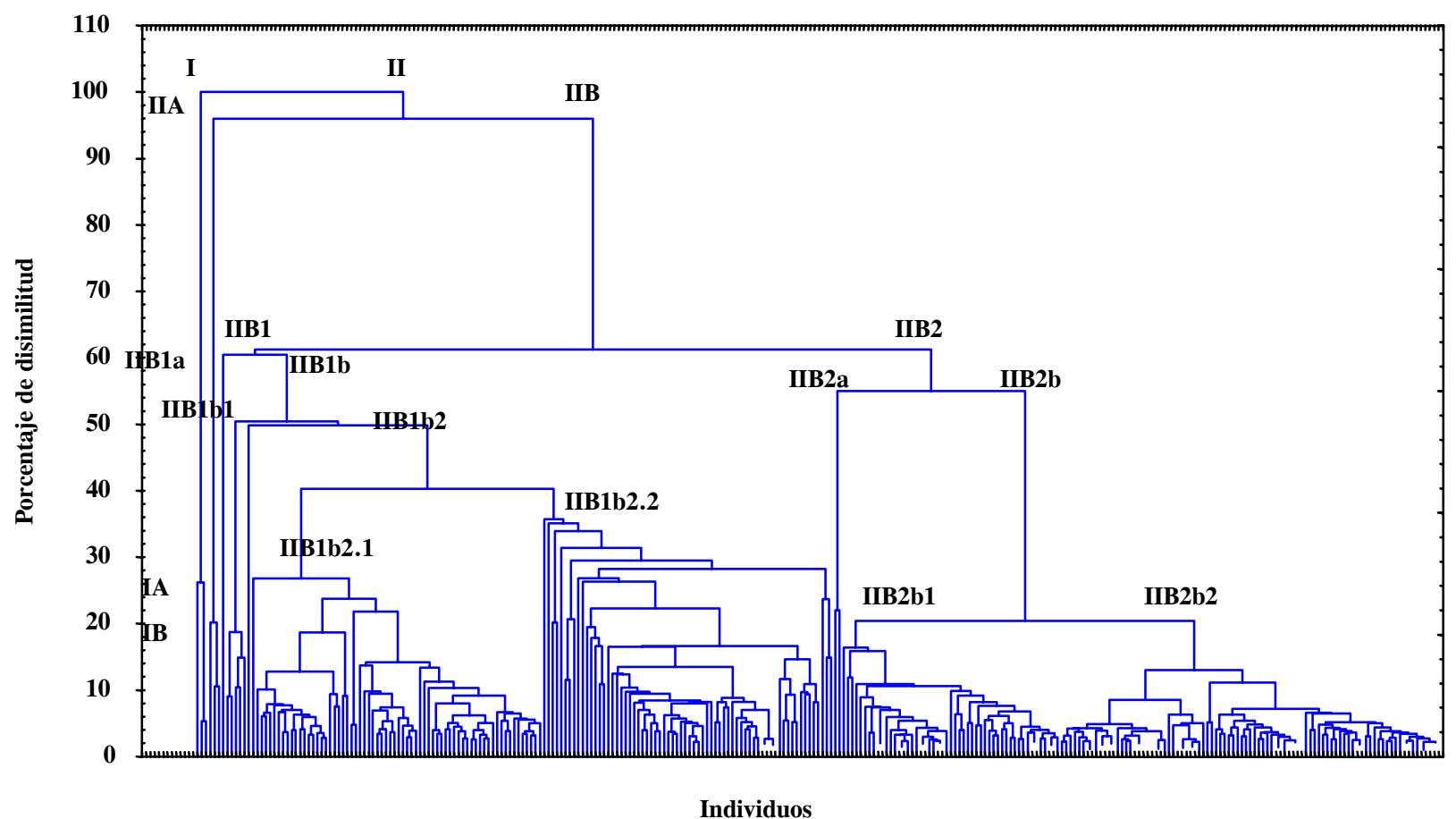

Figura 1. Dendrograma de disimilitudes de 219 árboles de Persea americana var. drymifolia del banco de germoplasma del INIFAP en Celaya, Gto. Cada línea que toca el eje de las $\mathrm{X}$ representa a un árbol.
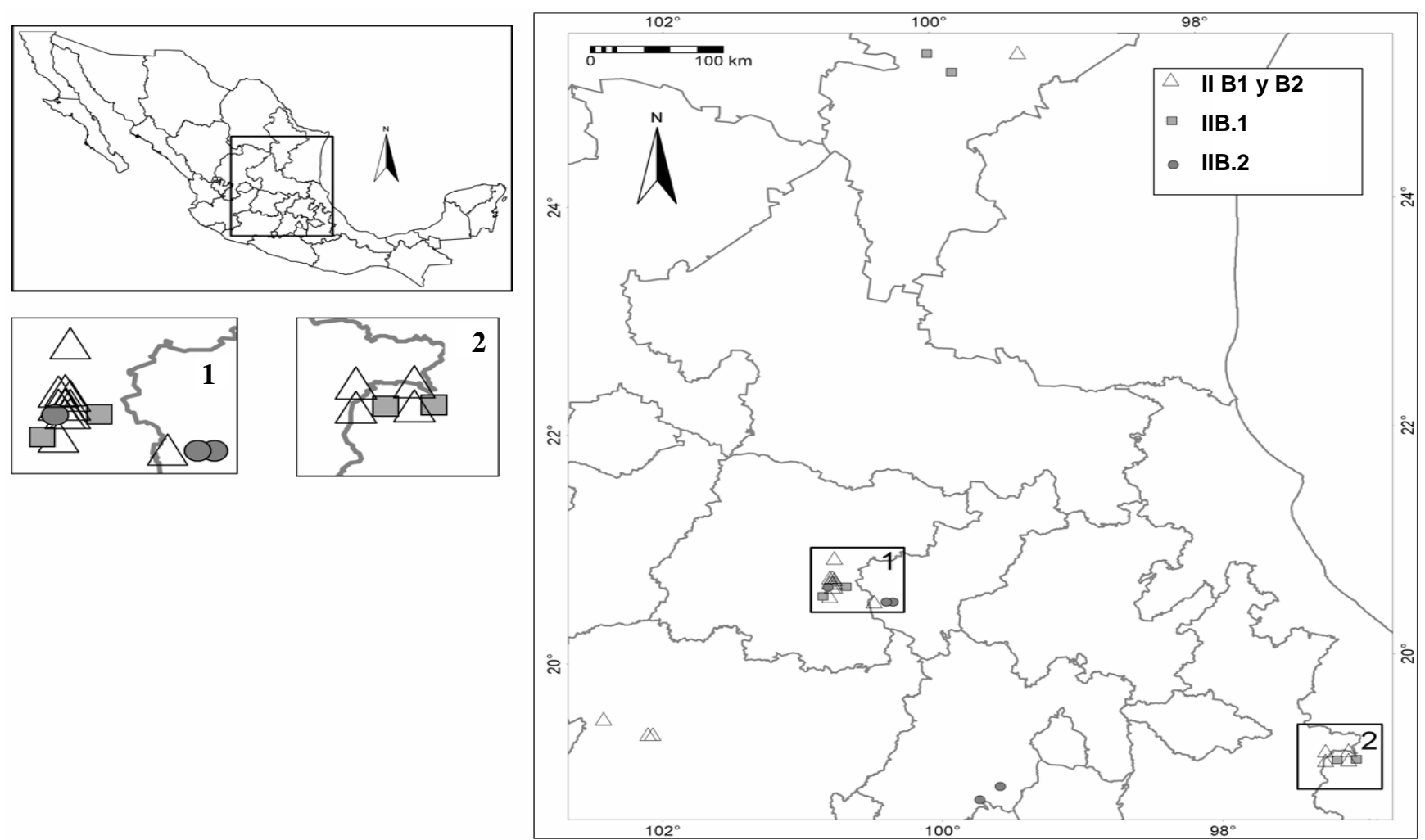

Figura 2. Mapa que presenta la distribución de los grupos de individuos IIB.1 y IIB.2, de Persea americana var. drymifolia relacionado con el diagrama de conglomerados. 

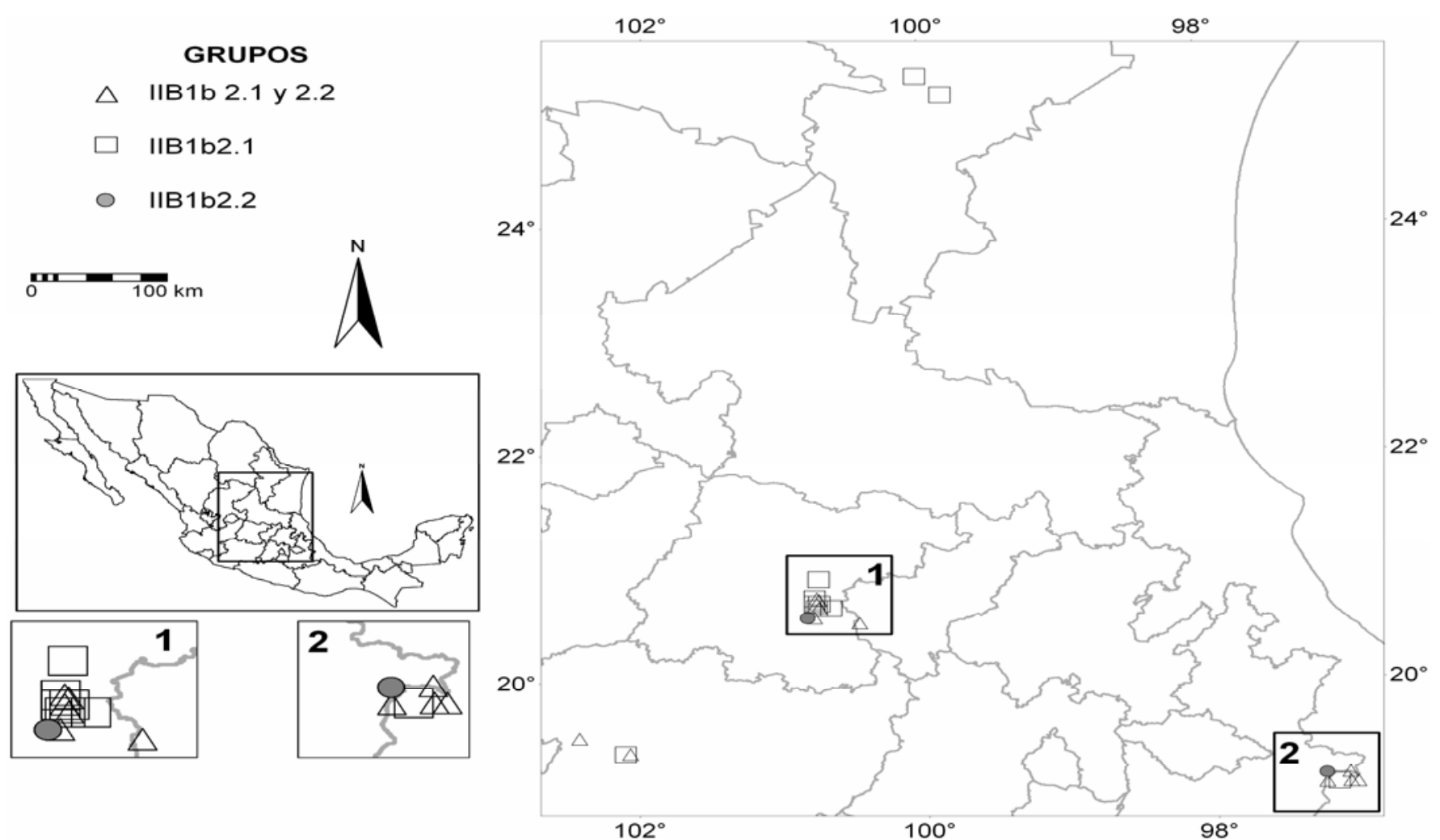

Figura 3. Mapa que presenta la distribución de los grupos de individuos IIB1b2.1 y IIB1b2.2 de Persea americana var. drymifolia, relacionado con el diagrama de conglomerados.

\section{GRUPOS}

$\triangle$ IIB $2 b .1$ y $2 b .2$

$\square$ IIB2b.1

○ IIB2b.2
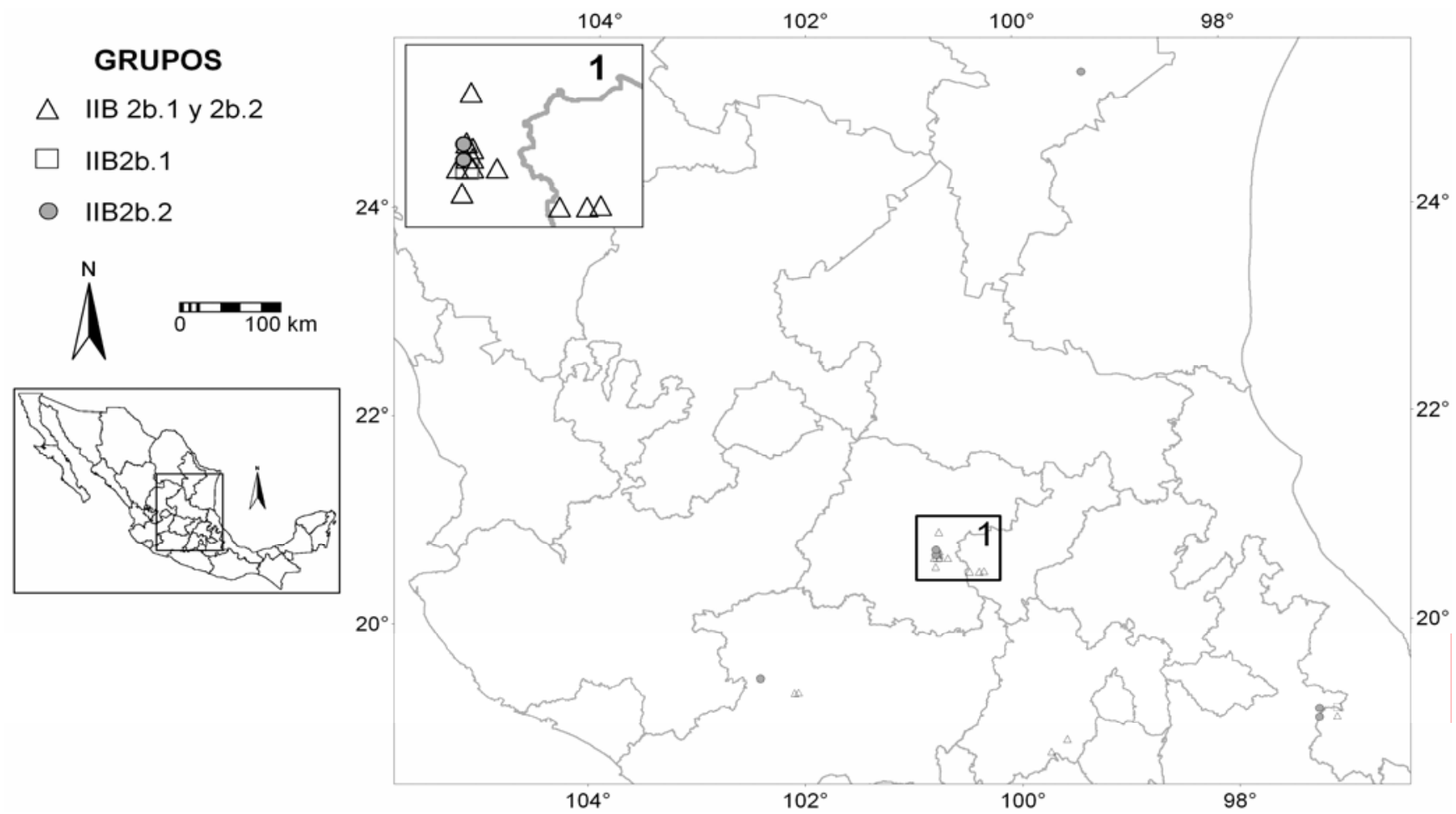

Figura 4. Mapa que presenta la distribución de los grupos de individuos IIB2b.1 y IIB2b.2 de Persea americana var. drymifolia, relacionado con el diagrama de conglomerados. 


\section{Variación química dentro y entre estados}

Tres a 19 localidades se anidaron dentro de cinco estados (Cuadro 2); el porcentaje de variación explicada fue mayor entre estados que dentro de cada estado (64 a $72 \%$ y 20 a $56 \%$, respectivamente). De estos resultados, $\beta$-pineno, cis- $\beta$-terpineol, 1c.n.i.(5.02), estragol, octadecano presentaron diferencias $(\mathrm{P} \leq 0.05)$ entre estados y dentro de ellos; $p$-cimeno, cariofileno, hexadecano y nerolidol sólo presentaron diferencias dentro de estados. En el resto de los compuestos no hubo diferencias ni entre ni dentro de estados.

\section{Proporción de compuestos más abundantes en las localidades}

En los compuestos más abundantes (Figura 5) se encontró que $\beta$-pineno fue mayor en General Terán, N. L. (13\%), y no se encontró en Calcahualco3, Ver. (Figura 5a); $p$-cimeno en Uruapan, Mich. tuvo el porcentaje más alto (13\%) y el más bajo en Calcahualco1, Ver. (2 \%) (Figura 5b); estragol fue el más abundante de todos los compuestos presentes, y su porcentaje más alto lo tuvieron Tetela del Volcán, Mor. (55 \%) y Tenancingo, Edo. de México (55\%), mientras que el menor porcentaje fue en Coscomatepec, Ver. (23 \%) (Figura 5c). El mayor porcentaje de eugenol-metil-éter lo presentó Calcahualco3, Ver. (8 \%) y el menor Villa Corregidora, Qro. (1 \%) (Figura 5d); cariofileno presentó un patrón muy estable entre 9 y $12 \%$, excepto en San Miguel de Allende, Gto. (16 \%) (Figura 5e); germacreno D observó el mayor porcentaje en Peribán de Ramos, Mich. (6\%) y el más bajo en Comonfort2, Gto. (1 \%) (Figura 5f); finalmente, hexadecano manifestó el valor más alto en Peribán de Ramos, Mich. (8 \%) y el más bajo en Calcahualco1, Ver. (1\%) (Figura 5g)

\section{Correlación entre la localización geográfica y la incidencia de los compuestos volátiles}

En tres de los análisis de regresión múltiple cuyas variables independientes fueron altitud, latitud y longitud (Cuadro 4), se produjeron modelos estadísticamente significativos para explicar la concentración de $\beta$-pineno, $p$ cimeno, estragol, eugenol-metil-éter y hexadecano, después de aplicar la corrección de Bonferroni. Sin embargo, las $\mathrm{R}^{2}$ de los modelos son muy bajas, por lo que posiblemente no sean de relevancia biológica.

No se encontró relación entre el aumento de la latitud y la concentración de los compuestos más abundantes de las localidades muestreadas en el banco de germoplasma (Figura 6). $\beta$-pineno fue en aumento hasta llegar a $19^{\circ} 23^{\text {' }}$ LN en Uruapan, Mich. (4 \%), y luego disminuyó y volvió a aumentar hasta los $25^{\circ} 20^{\prime}$ LN en General Terán, N.L. (6\%). Cariofileno aumentó y disminuyó constantemente a lo largo del gradiente, con un valor máximo en General Terán (13\%); p-cimeno tuvo el mismo patrón que el anterior, con su valor más alto a $19^{\circ} 23^{\prime} \mathrm{LN}$ en Uruapan (5\%), después disminuyó y se estabilizó en General Terán $(3 \%)$. Hexadecano presentó el mismo patrón anterior, con el valor más alto a $19^{\circ} 31^{\prime}$ LN en Peribán de Ramos, Mich. (3\%). Eugenol-metil-éter, estragol y germacreno D no mostraron patrón alguno, aunque se observó variación a lo largo del gradiente latitudinal (Figura 6).

Cuadro 4. Resumen del análisis de regresión linear múltiple para los compuestos foliares más abundantes encontrados en los árboles de $P$. americana var. drymifolia del Banco de Germoplasma INIFAP en Celaya, Guanajuato.

\begin{tabular}{|c|c|c|c|}
\hline Compuestos & $\begin{array}{c}\text { Factor de } \\
\text { ubicación geográfica }\end{array}$ & Beta & $t(287)$ \\
\hline \multirow[t]{2}{*}{$\beta$-pineno: $\mathrm{R}=0.2, \mathrm{~F}(2,288)=7.1$, Error estándar del estimado $=4.3$} & Longitud & $0.2 \pm 0.1$ & $2.9 * *$ \\
\hline & Altitud & $-0.1 \pm 0.1$ & $-2.5^{*}$ \\
\hline \multicolumn{4}{|l|}{$p$-cimeno: $\mathrm{R}=0.2, \mathrm{~F}(1,289)=12.2$, Error estándar del estimado $=$} \\
\hline 5.0 & Longitud & $0.2 \pm 0.1$ & $3.5^{* * *}$ \\
\hline $\begin{array}{l}\text { Estragol: } \mathrm{R}=0.3, \mathrm{~F}(3,287)=13.1, \text { Error estándar del estimado }= \\
20.3\end{array}$ & Longitud & $0.2 \pm 0.1$ & $2.3^{*}$ \\
\hline \multirow{2}{*}{$\begin{array}{l}\text { Eugenol-metil-éter: } \mathrm{R}=0.2, \mathrm{~F}(2,288)=7.9 \text {, Error estándar del esti- } \\
\text { mado }=6.6\end{array}$} & Longitud & $-0.2 \pm 0.1$ & $-3.5 * * *$ \\
\hline & Altitud & $-0.1 \pm 0.1$ & $-1.9 \mathrm{~ns}$ \\
\hline \multirow{2}{*}{$\begin{array}{l}\text { germacreno } \mathrm{D}: \mathrm{R}=0.1, \mathrm{~F}(2,288)=1.4 \text {, Error estándar del estima- } \\
\mathrm{do}=2.9\end{array}$} & Longitud & $0.1 \pm 0.1$ & $1.2 \mathrm{~ns}$ \\
\hline & Altitud & $-0.1 \pm 0.1$ & $-1.2 \mathrm{~ns}$ \\
\hline
\end{tabular}


El banco de germoplasma mostró alta variación en los perfiles químicos de $P$. americana var. drymifolia, manifestada en árboles que crecieron en el ambiente del banco de germoplasma; esto sugiere que los perfiles están determinados genéticamente. La presencia y concentración de estragol, compuesto mayoritario en aguacate criollo, tiene base genética. Los terpenos están bajo un fuerte control genético en otras especies arbóreas ( Gershenzon et al., 2000; Langenheim y Stubblebine, 1983; McConkey et al., 2000), y es posible que también lo estén en el aguacate criollo, de acuerdo con la variación manifestada en un ambiente común representado por el banco de germoplasma. Más aún, muchas especies muestran diferencias genéticas en la formación de terpenoides (Mihaliak et al., 1989; Mithen et al., 1995; Shonle y Bergelson, 2000). Por ello aquí se postula que las diferencias químicas encontradas en el aguacate criollo son una buena representación de la variación genética de esta variedad de aguacate.

La mayor parte de los árboles de aguacate quedaron agrupados en conjuntos de fenotipos químicos con 40 a 80 $\%$ de similitud, sin diferencias significativas en la distribución geográfica de las accesiones (Figuras 2 a 4). Esto indica que dentro de las localidades de origen y entre los estados hay variación alta que posiblemente se originó tempranamente en la historia de la diseminación del aguacate criollo. No obstante, también se encontró amplia variación y diferenciación entre las localidades de origen del aguacate criollo y los estados donde se ubican (Cuadro 2; Figura 6). La mayor variación entre estados que dentro de ellos sugiere diferenciación genética, que coincide con lo encontrado en $P$. americana donde la razas antillana, guatemalteca y mexicana difieren química y genéticamente entre sí (Bergh et al., 1973); también es similar a lo encontrado en muchas coníferas (Dodd y Rafii, 1994; Nava-Cruz et al., 2006; Naydenov et al., 2005). Pero es contrario a lo encontrado en otras coníferas ( Rafii et al., 1992; Rudloff y Lapp, 1989; Zavarin et al., 1976), y en la laurácea Umbellularia californica (Goralka y Langenheim, 1995) donde la variación mayor dentro de las poblaciones se atribuye a un alto flujo génico entre ellas.

La variación en los perfiles químicos promedio de las localidades estudiadas (Figura 6), sugiere diferenciación posiblemente reciente que podría estar relacionada con el conjunto de herbívoros y patógenos propios de cada localidad. Una diferenciación de este tipo se ha encontrado en los terpenos de Pinus ponderosa, donde los herbívoros determinan perfiles químicos de las poblaciones (Sturgeon, 1979). En cada población de aguacate se observó un fenotipo químico promedio diferente (Figura 6), en donde los compuestos más concentrados difieren entre poblaciones (Figura 5). Estas diferencias químicas pueden ser determinantes en interacciones bióticas; por ejemplo, los pinos con mayor concentración de $\beta$-pineno son menos atacados que los que contienen menor cantidad de este compuesto (Snyder, 1992), al igual que ocurre en otras especies de pinos (Zavarin et al., 1990 , 1993). El fenotipo químico promedio de cada población probablemente está determinado por su ambiente biótico particular y refleja un perfil químico adaptado a este ambiente. Los resultados muestran mosaicos geográficos de interacciones bióticas, las que a su vez pueden estar determinadas por metabolitos secundarios (Thompson, 2005), lo que explicaría la variación en los fenotipos químicos promedio de las localidades.

A escala individual, el fenotipo químico determina en muchas ocasiones la susceptibilidad del individuo a herbívoros y patógenos ( Edwards et al., 1993; EspinosaGarcía et al., 2001; Langenheim, 2003); en la interacción aguacate criollo-Trioza los árboles con más concentración de estragol tienen menos agallas foliares (RincónHernández y Espinosa-García, 2008).

En el presente estudio el estragol es el único compuesto que presentó relación significativa positiva con la altitud, latitud y longitud geográficas, mientras que el $\beta$ pineno, $p$-cimeno, estragol, hexadecano y eugenol-metiléter se relacionaron solamente con la longitud; los primeros cuatro se relacionan de forma positiva y el último en forma negativa (Cuadro 4). Esto no es sorpresivo pues la incidencia de varios terpenoides está relacionada con la altitud, latitud y longitud en algunas especies de pináceas (Hengxiao et al., 1999; Lockhart, 1990), lamiáceas (Azevedo et al., 2001) y asteráceas (Zidorn y Stuppner, 2001). También se ha encontrado que la incidencia de alcaloides se relaciona con la altitud y latitud (Levin, 1976; Moody, 1978). Estos gradientes geográficos en la abundancia de los metabolitos secundarios se han interpretado en función de la intensidad del gradiente de presión de selección ejercido por herbívoros y patógenos (Levin y York, 1978; Salmore y Hunter, 2001). Para los compuestos del aguacate criollo que variaron en gradientes, es posible que la presión de herbívoros y patógenos estén correlacionadas con estos compuestos, aunque aún no hay evidencia contundente de ello (Rincón-Hernández y Espinosa-García, 2008).

Puesto que el estado de conservación de bosques de $P$. americana var. drymifolia es muy precario (Challenger, 1998; Lorea-Hernández, 2002), la conservación de la variación genética de esta especie al menos en bancos de germoplasma resulta crucial. El banco de germoplasma estudiado concentra genotipos de diferentes lugares en donde probablemente ya no existan individuos de estas 
poblaciones. Más aún, también conserva una amplia variación química que representa a los estados del centro del país, incluido el eje neovolcánico donde se cree que se originó esta variedad de aguacate (Barrientos-Priego y López-López, 1998). El Banco de Germoplasma del INIFAP- Celaya debería de seguir siendo conservado y protegido. Estos resultados también muestran que hay diferenciación significativa entre aguacates criollos de diferentes estados, por lo que sería importante que si no se
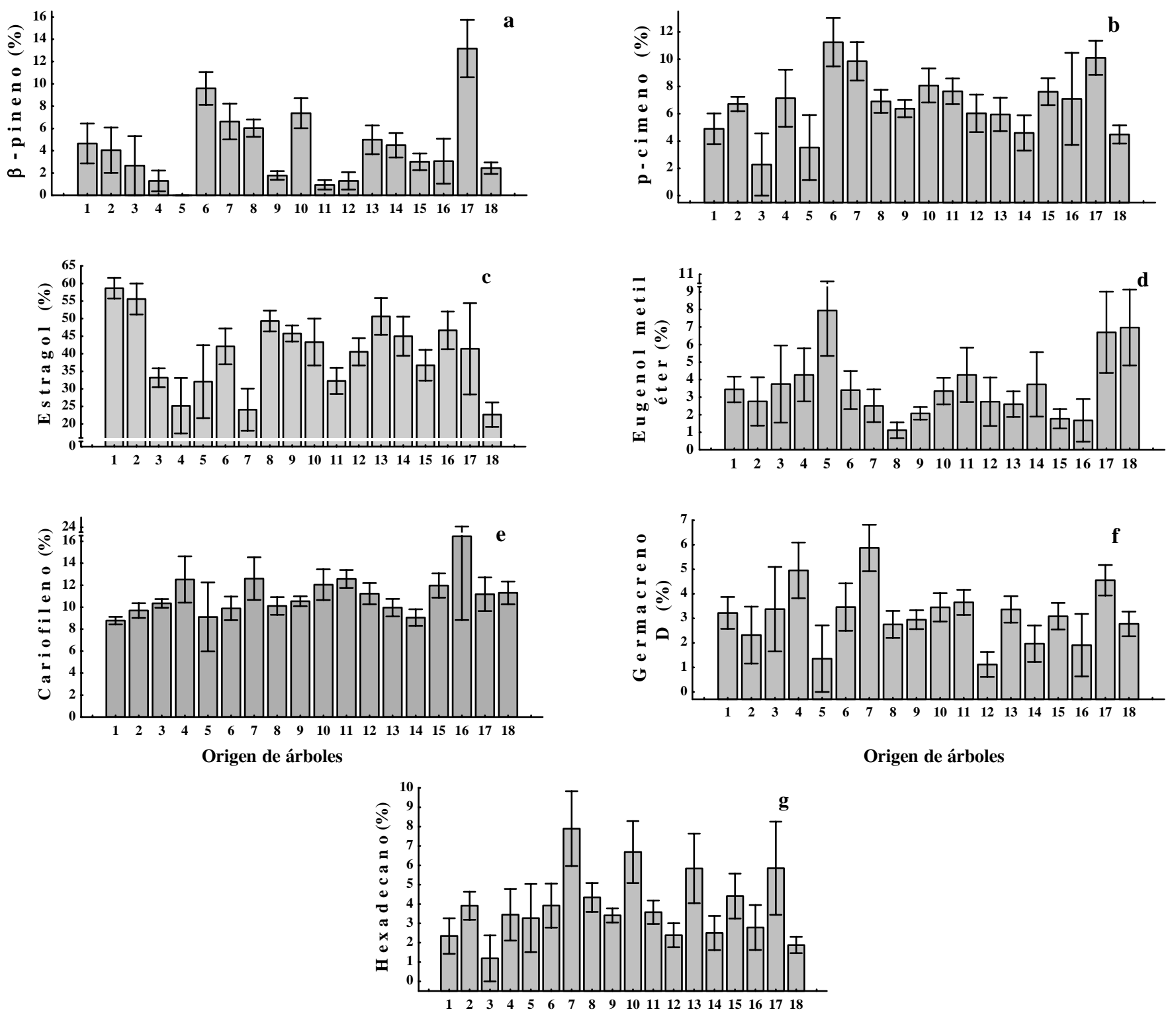

Origen de árboles

Figura 5. Porcentajes promedio de los compuestos más abundantes en hojas de 286 árboles de Persea americana var. drymifolia, con su respectivo error estándar. Los números en el eje de las X representan: 1, Tetela del Volcán, Mor.; 2, Tenancingo, Edo. de México; 3, Calcahualco1, Ver.; 4, Calcahualco2, Ver.; 5, Calcahualco3, Ver.; 6, Uruapan, Mich.; 7, Peribán de Ramos, Mich.; 8, Villa Corregidora, Qro.; 9, Celaya1, Gto.; 10, Celaya2, Gto.; 11, Comonfort1, Gto.; 12, Comonfort2, Gto.; 13, Comonfort3, Gto., 14, Comonfort4, Gto.; 15, Comonfort5, Gto.; 16 San Miguel de Allende, Gto.; 17, General Terán, N.L. y 18 Coscomatepec, Ver. (a) $\beta$-pineno, (b) p-cimeno, (c) estragol, (d) eugenol metil éter, (e) cariofileno, (f) germacreno D y (g) hexadecano. 


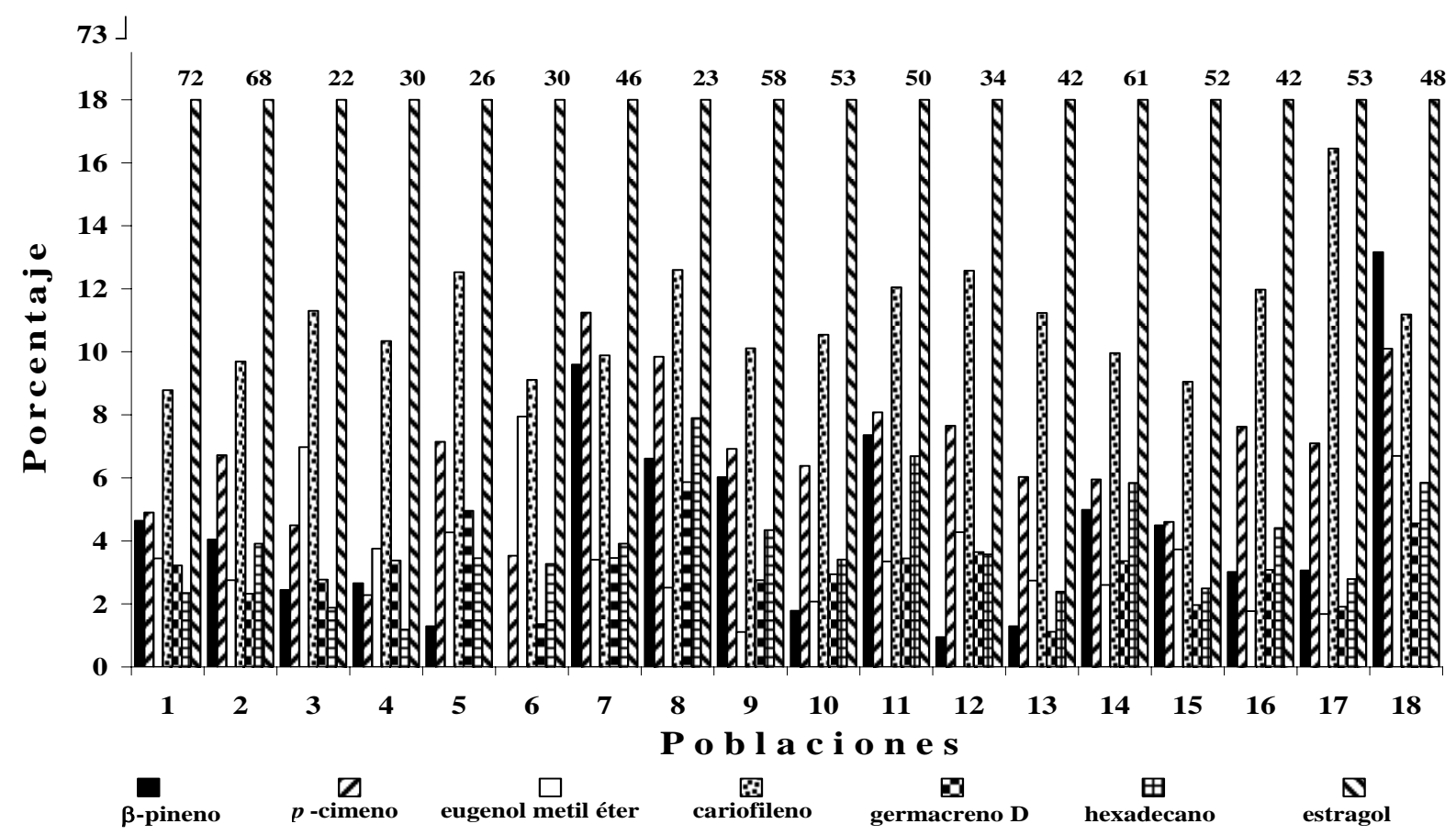

Figura 6. Porcentaje de compuestos en hojas de Persea americana var. drymifolia provenientes de 18 localidades con un gradiente latitudinal de $1^{\circ} 4^{\prime}$ ' LN en la localidad 1 hasta $2^{\circ} 20^{\prime}$ LN en la localidad 18. Los números en el eje de las $X$ representan con: 1, Tetela del Volcán, Mor. 2, Tenancingo, Edo. de Méx. 3, Coscomatepec, Ver. 4, Calcahualco1, Ver. 5, Calcahualco2, Ver. 6, Calcahualco3, Ver. 7, Uruapan, Mich. 8, Peribán de Ramos, Mich. 9, Villa Corregidora, Qro. 10, Celaya1, Gto. 11, Celaya2, Gto. 12, Comonfort1, Gto. 13, Comonfort2, Gto. 14, Comonfort3, Gto. 15, Comonfort4, Gto. 16, Comonfort5, Gto. 17 San Miguel de Allende, Gto. y 18, General Terán, N.L.

Estos resultados sugieren que las áreas de distribución natural del aguacate criollo en México, que no están representadas en el banco de germoplasma de INIFAP, poseen fenotipos químicos únicos que deberían de conservarse en éste $u$ otros bancos de germoplasma.

\section{CONCLUSIONES}

El aguacate criollo es un taxón muy variable químicamente, posiblemente debido a su variación genética. Esta variación química podría ser importante para identificar árboles con fenotipos químicos relevantes y como fuente de genes de resistencia contra plagas y enfermedades de variedades comerciales de aguacate.

Los compuestos predominantes en Persea americana var. drymifolia fueron estragol (22 a $72 \%)$ y cariofileno (9 a $16 \%$ ), pero la abundancia de éstos y los otros compuestos en los árboles varían de acuerdo con su localidad de origen.

\section{AGRADECIMIENTOS}

Al Centro de Investigaciones en Ecosistemas, UNAM (POFJEG) y al Consejo Nacional de Ciencia y Tecnología (Proyecto 34895-V otorgado a FJEG), por el apoyo económico. Al geógrafo Jesús Fuentes Junco, por la elabora- ción de los mapas. A la Biól. Yolanda García, R., Biól. Ángel Bravo M. y M. C. Heberto Ferreira por su ayuda técnica. Al CONACyT por la beca que otorgó a la primera autora durante sus estudios de doctorado en Ciencias Biomédicas de la UNAM.

\section{BIBLIOGRAFÍA}

Azevedo N R, I F P Campos, H D Ferreira, T A Portes, S C Santos, J C Seraphin, J R Paula, P H Ferri (2001) Chemical variability in the essential oil of Hyptis suaveolens. Phytochemistry 57:733-736.

Barrientos-Priego A F, L López-López (1998) Historia y genética del aguacate. In: Memoria Fundación Salvador Sánchez Colin. CICTAMEX S.C. Coatepec Harinas, México. pp:33-51.

Bergh B O, R W Scora, W B Storey (1973) A comparison of leaf terpenes in Persea Subgenus Persea. Bot. Gaz. 134:130-134.

Challenger A (1998) La zona ecológica templada húmeda (bosque mesófilo de montaña). In: Utilización y Conservación de los Ecosistemas Terrestres de México. Pasado, Presente y Futuro. T Segovia, G Lara (eds). CONABIO. Instituto de Biología, UNAM. Agrupación Sierra Madre, S.C. México, D.F. pp:443-518.

Dodd R S, Z A Rafii (1994) Chemical and ecological variability of $\mathrm{Cu}$ pressus bakeri on Goosenest Mountain, California. Biochem. Syst. Ecol. 22:393-400.

Edwards P B, W J Wanjura, W V Brown (1993) Selective herbivory by Christmas beetles in response to intraspecific variation in Eucalyptus terpenoids. Oecologia 95:551-557.

Espinosa-García F J (2001) La diversidad de los metabolitos secundarios y la teoría de la defensa vegetal. In: Relaciones Químicas entre Organismos. Aspectos Básicos y Perspectivas de su 
Aplicación. A L Anaya, F J Espinosa-García, R Cruz-Ortega (eds). Universidad Nacional Autónoma de México y Plaza y Valdés. México, D.F. pp:231-249.

Espinosa-García, F J, Y García-Rodríguez, F Chávez-Zavala, A Chávez-Zavala, G Delgado (2001) Implicaciones de la variación en los fenotipos químicos de las poblaciones de plantas en su susceptibilidad a plagas y patógenos: el caso de Persea americana var. Hass In: Ier. Congreso Mexicano y Latinoamericano del Aguacate. Uruapan, Mich., México pp:4657

FAO-UNESCO (1970) Clave de Unidades de Suelos para el Mapa de Suelos del Mundo. Traducción de E. García A. 1974. Dirección de Agrología. Secretaria de Recursos Hidraúlicos.

Fielder J, G Bufler, F Bangerth (1998) Genetic relationships of avocado (Persea americana Mill.) using RAPD markers. Euphytica 101:249-255.

García E (1988) Modificaciones al Sistema de Clasificación Climática de Köppen (para adaptarla a las condiciones de la República Mexicana).

Gershenzon J, M E McConkey, R B Croteau (2000) Regulation of monoterpene accumulation in leaves of peppermint. Plant Physiol. 122:205-213.

Goralka R J L, J H Langenheim (1995) Analysis of foliar monoterpenoid content in the California Bay Tree, Umbellularia californica among populations across the distributions of the species. Biochem. Syst. Ecol. 23:439-448.

Hengxiao G, J D McMillin, M R Wagner, J Zhou, Z Zhou, X Xu (1999) Altitudinal variation in foliar chemistry and anatomy of yunnan pine, Pinus yunnanensis, and pine sawfly (Hym., Diprionidae) performance. J. Appl. Entomol. 123:465-471.

Langenheim J H (2003) Ecological roles of resins. In: Plant Resins: Chemistry, Evolution, Ecology and Ethnobotany. Timber Press Science. Portland, Oregon USA. pp:196-253.

Langenheim J H, W H Stubblebine (1983) Variation in leaf resin composition between parent tree and progeny in Hymenaea: Implications for herbivory in the humid tropics. Biochem. Syst. Ecol. 11:97-106.

Lavi U, E Lahav, C Degani, S Gazit (1993a) Genetics of skin solor, flowering group and anise scent in avocado. J. Hered. 84:8284.

Lavi U, E Lahav, C Degani, S Gazit, J Hillel (1993b) Genetic variance components and heritabilities of several avocado traits J. Am. Soc. Hortic. Sci. 118:400-404.

Levin D A (1976) Alkaloid-bearing plants: an ecogeographic perspective. Am. Nat. 110:261-284.

Levin D A, B M York (1978) The toxicity of plant alkaloids: An ecogeographic perspective. Biochem. Syst. Ecol. 6:61-76.

Lockhart L A (1990) Chemotaxonomic relationships within the Central American closed-cone pines. Silvae Genet. 39:173-184.

Lorea-Hernández F G (2002) La familia Lauraceae en el sur de México: diversidad, distribución y estado de conservasión. Bol. Soc. Bot. Méx. 71:59-70.

McConkey M E, J Gershenzon, R B Croteau (2000) Developmental regulation of monoterpene biosynthesis in the glandular trichomes of peppermint. Plant Physiol. 122:215-223.

Mhameed S, J Hillel, E Lahav, D Sharon, U Lavi (1995) Genetic association between DNA fingerprint fragments and loci controlling agriculturally important traits in avocado (Persea americana Mill.). Euphytica 84:81-87.

Mihaliak C A, D Couvet, D E Lincoln (1989) Genetic and environmental contributions to variation in leaf mono- and sesquiterpenes of Heterotheca subaxillaris. Biochem. Syst. Ecol. 17:529-533.

Mithen R, A F Raybould, A Giamoustaris (1995) Divergent selection for secondary metabolites between wild populations of Brassica oleracea and its implications for plant-herbivore interactions. Heredity 75:472-484.
Moody S (1978) Latitude, continental drift, and the percentage of alkaloid-bearing plants in floras. Am. Nat. 112:965-968

Nava-Cruz Y, F J Espinosa-García, G R Furnier-Whitelaw (2006) Levels and patterns of chemical variation in leaf resins of the genus Abies from northern México. Agrociencia 40:229-238.

Naydenov K D, F M Tremblay, Y Bergeron, A Alexandrov, N Fenton (2005) Dissimilar patterns of Pinus heldreichii Christ. populations in Bulgaria revealed by chloroplast microsatellites and terpenes analysis. Biochem. Syst. Ecol. 33:133-148.

Prabha T N, B Ravindranath, M V Patwrdhan (1980) Anthocyanins of avocado (Persea americana) peel. J. Food Sci. Tech. 17:241-242.

Rafii A Z, L G Cool, E Zavarin (1992) Variability of foliar mono- and sesquiterpenes of Cupressus bakeri. Biochem. Syst. Ecol. 20:123-131.

Rincón-Hernández, C, J L Sánchez Pérez, F J Espinosa-García (2005) Caracterización química de los árboles de Persea americana Mill. var. drymifolia (Schltdl. \& Cham.) S.F. Blake de los bancos de germoplasma INIFAP (Michoacán). In: Memorias del II Congreso Mexicano y Latinoamericano del Aguacate. Uruapan, Mich., México. de 17 al 21 de octubre pp:1-7.

Rincón-Hernández C A, F J Espinosa-García (2008) Relationship between the phytochemical diversity in creole avocado (Persea americana var. drymifolia) leaves and the incidence of foliar gall psyllid (Trioza anceps). Allelopathy J. 21:175-182.

Rudloff E v, M S Lapp (1989) Some observations on the leaf oil terpene composition of mountain hemlock, Tsuga mertensiana. Can. J. Forest Res. 19:848-852.

Sagrero-Nieves L, J P Bartley (1995) Volatile components of avocado leaves (Persea americana Mill) from the Mexican race. J. Sci. Food Agr. 67:49-51.

Salmore A K, M D Hunter (2001) Elevational trends in defense chemistry, vegetation, and reproduction in Sanguinaria canadensis. J. Chem. Ecol. 27:1713-1727.

Sánchez-Pérez J L (1999) Recursos genéticos de aguacate (Persea americana Mill.) y especies afines en México. Rev. Chapingo $\mathrm{S}$. Hort. 5:7-18.

Shonle I, J Bergelson (2000) Evolutionay ecology of the tropane alkaloids of Datura stramonium L. (Solanaceae). Evolution 54:778-788.

Snyder M A (1992) Selective herbivory by Albert's squirrel mediated by chemical variability in ponderosa pine. Ecology 73:17301741.

Sturgeon K B (1979) Monoterpene variation in ponderosa pine xylem resin related to western pine beetle predation. Evolution 33:803-814

Thompson J N (2005) Local adaptation I. Geographic selection mosaics. In: The Geographic Mosaic of Coevolution. The University of Chicago. USA. pp:50-71.

White E E, J E Nilsson (1984) Foliar terpene heritability in Pinus contorta. Silvae Genet. 33:16-22.

Williams L O (1977) The avocados, a synopsis of the genus Persea subg. Persea. Econ. Bot. 31:315-320.

Zavarin E, L G Cool, K Snajberk (1993) Geographic variability of Pinus flexilis xylem monoterpenes. Biochem. Syst. Ecol. 21:381-387.

Zavarin E, K Snajberk, D Bailey (1976) Variability in the essential oils of wood and foliage of Pinus aristata and Pinus logaeva. Biochem. Syst. Ecol. 4:81-92.

Zavarin E, K Snajberk, L Cool (1990) Monoterpene variability of Pinus monticola wood. Biochem. Syst. Ecol. 18:117-124.

Zidorn C, H Stuppner (2001) Evaluation of chemosystematic characters in the genus Leontodon. Taxon 50:115-133. 